\title{
Rigid Curves at Random Positions and Linking Numbers
}

\author{
J. des Cloizeaux and R. Ball
}

Service de Physique Théorique, CEN-Saclay, Boite Postale No 2, F-91190 Gif-sur-Yvette, France

\begin{abstract}
A property of the square of the linking number of two closed rigid curves randomly displaced in a three dimensional space, has been recently found by $\mathrm{W}$. Pohl. Here, this result is reproduced and generalized. This new approach is quite different and uses a simple Fourier transformation.
\end{abstract}

\section{Introduction}

The theory of knots is not only very interesting from a mathematical point of view, it may also have fruitful applications in polymer physics.

A polymer molecule consists of a long sequence of chemically connected units, each of which comprises only a few atoms. The long chains so formed can be quite flexible and for many purposes their behaviour can be modelled by representing them simply by smooth mathematical curves embedded in space (i.e. $R^{3}$ or $S^{3}$ ). For instance consider two polymer molecules each of which has the connectivity of a loop (i.e. $S^{1}$ ). In their physical motion, they may move and deform in a continuous fashion but not cross through each other or themselves. At the level of mathematical curves of zero thickness, this is equivalent to saying that they may not pass through configurations with double points. Thus the two molecules conserve their topology and they can physically be separated if and only if their configuration is not topologically linked.

No prescription can be given for classifying completely the linking of two loops. A very simple and versatile partial description is given by Gauss' "linking number" (or "winding number"). Roughly, this quantity is the number of turns one curve winds around the other and vice versa. More precisely we must first define a sense of direction around each of the two curves. Then choose an oriented surface whose boundary is one curve and count algebraically the total number of intersections of the second curve with this surface, \pm 1 according to whether the surface is approached from its \pm side by the second curve at each intersection. 
Note that all topologically equivalent links have the same linking number (up to a possible difference in sign if the senses of direction are not preserved), but inequivalent ones will not necessarily be distinguished by it. All unlinked (i.e. separable) configurations have linking number zero, but so also do many topologically linked ones.

Rolfsen [1] gives many more equivalent definitions of the linking number, but of particular interest here is the integral [2] given by Gauss for this integer-valued topological invariant:

$$
I_{A B}=\frac{1}{4 \pi} \int_{A} \int_{B} \frac{\left(d \mathbf{r}_{A}, d \mathbf{r}_{B}, \mathbf{r}_{A B}\right)}{r_{A B}^{3}} .
$$

Here the vector $\mathbf{r}_{A}$ defines a point on curve $A$, the vector $\mathbf{r}_{B}$ a point on curve $B$ and $\mathbf{r}_{A B}=\mathbf{r}_{B}-\mathbf{r}_{A}$. The integrations are performed along each curve ${ }^{1}$ and $I_{A B}$ changes sign upon changing the sense of direction of either curve.

W. Pohl recently found a new result [3] concerning the square of the linking number of two curves, which may be very useful in the polymer applications. Trying to reproduce this result, we found a new simple derivation and a generalized version of Pohl's formula. The aim of this article is to present this new approach which uses Fourier transforms.

Let us take some initially given joint configuration of the curves $A$ and $B$. Then by translating curve $B$ with respect to curve $A$ by a vector $\rho$ and by changing the orientation $\mathcal{O}$ of $B$ with respect to $A$, we can define the linking number $I_{A B}(\rho, \mathcal{O})$.

It is not difficult to show that

$$
\int d^{3} \rho\left\langle I_{A B}(\rho, \mathcal{O})\right\rangle=0,
$$

where the brackets indicate that we average over all orientations $\mathcal{O}$ of $A$ and $B$. However, it is more interesting to calculate the second moment,

$$
J_{A B}=\int d^{3} \rho\left\langle I_{A B}^{2}(\rho, \mathcal{O})\right\rangle \text {. }
$$

We note that for plane convex curves $I_{A B}^{2}$ is always one or zero. In this case, $J_{A B}$ has a simple physical interpretation. It defines the "excluded volume" of the curves $A$ and $B$. Thus the second virial coefficient of a gas (or a solution) of convex planar curves $C$ is given directly by $\frac{1}{2} J_{C C}$.

Using a slightly different notation, W. Pohl has shown, for plane convex curves, that $J_{A B}$ can be written in the form

$$
J_{A B}=\frac{1}{8 \pi} \int_{0}^{\infty} d r \mathscr{A}_{A}(r) \mathscr{A}_{B}(r)
$$

where $\mathscr{A}_{A}(r)$ and $\mathscr{A}_{B}(r)$ are functions associated with the curves $A$ and $B$. As was shown by W. Pohl, for a closed curve, the function $\mathscr{A}(r)$ is defined by the following integral taken along the curve (see Fig. 1)

$$
\mathscr{A}(r)=\int_{r_{12}=r} d s_{1} \cos \theta_{1}
$$

1 In (1.1), we use the notation $(\mathbf{a}, \mathbf{b}, \mathbf{c}) \equiv(\mathbf{a} \wedge \mathbf{b}) \cdot \mathbf{c}$ 

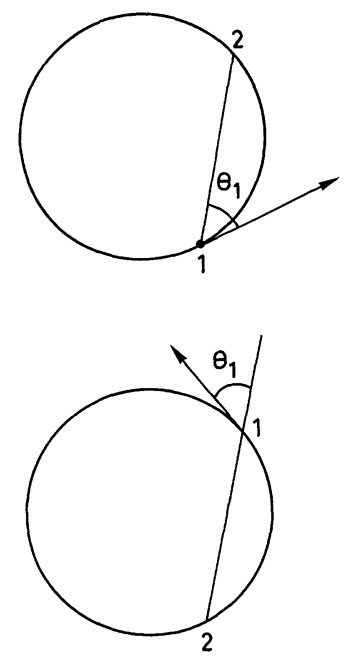

Fig. 1. For calculating $\mathscr{A}(r)$, the length $r_{12}=\left|\mathbf{r}_{1}-\mathbf{r}_{2}\right|$ is kept equal to the constant $r$

It is not difficult to show that for a plane convex curve, $\mathscr{A}(r)$ is just the length of the envelope of the segments of length $r$ joining two points 1 and 2 belonging to the curve. Thus for a circle of radius $R$

$$
\mathscr{A}(r)=2 \pi\left(4 R^{2}-r^{2}\right)^{1 / 2}
$$

On the other hand, for any curve of length $L$

$$
\mathscr{A}(0)=2 L \text {. }
$$

W. Pohl has indicated that his result can be extended to more complicated curves.

Using the approach described below, we found that in general

$$
J_{A B}=\frac{1}{8 \pi} \int_{0}^{\infty} d r\left[\mathscr{A}_{A}(r) \mathscr{A}_{B}(r)+\mathscr{B}_{A}(r) \mathscr{B}_{B}(r)\right]
$$

For a curve $C, \mathscr{A}(r)$ and $\mathscr{B}(r)$ are given by double integrals taken along $C$ :

$$
\begin{aligned}
& \mathscr{A}(r)=\frac{1}{r} \iint\left(d \mathbf{r}_{1} \cdot d \mathbf{r}_{2}\right) \theta\left(r-r_{12}\right) \\
& \mathscr{B}(r)=\frac{1}{r^{2}} \iint\left(d \mathbf{r}_{1}, d \mathbf{r}_{2}, \mathbf{r}_{12}\right) \theta\left(r-r_{12}\right),
\end{aligned}
$$

where $\theta(x)$ is the step function $\theta(x)=\frac{1}{2}(1+|x| / x)$ and $r_{12}=\left|\mathbf{r}_{2}-\mathbf{r}_{1}\right|$.

The function $\mathscr{B}(r)$ vanishes if $C$ is the same as its mirror image $C^{\prime}$ (obtained by reflection with respect to a plane or a point). Thus, $\mathscr{B}(r)=0$ for plane curves, but it will be shown in Sect. 4 that, in general, $\mathscr{B}(r)$ does not vanish. 

have

The expression for $\mathscr{A}(r)$ can be transformed by integrating by parts. We

$$
\begin{aligned}
\mathscr{A}(r) & =\frac{1}{r} \iint d \mathbf{r}_{1} \cdot d_{2}\left(\mathbf{r}_{2}-\mathbf{r}_{1}\right) \theta\left(r-r_{12}\right) \\
& =-\frac{1}{r} \iint\left(d \mathbf{r}_{1} \cdot \mathbf{r}_{12}\right) d_{2} \theta\left(r-r_{12}\right) .
\end{aligned}
$$

As $r_{12} d r_{12}=\left(\mathbf{r}_{12} \cdot d \mathbf{r}_{12}\right)$, we obtain for $\mathscr{A}(r)$ another form

$$
\mathscr{A}(r)=\frac{1}{r^{2}} \iint\left(d \mathbf{r}_{1} \cdot \mathbf{r}_{12}\right)\left(d \mathbf{r}_{2} \cdot \mathbf{r}_{12}\right) \delta\left(r-r_{12}\right)
$$

which is usually more convenient. In Eq. (1.10), the integration with respect to point 2 can be performed. Noting that

$$
\begin{aligned}
d \mathbf{r}_{1} \cdot \mathbf{r}_{12} & =\cos \theta_{1} r_{12} d r_{12} \\
d r_{12} & =\cos \theta_{2} d s_{2}-\cos \theta_{1} d s_{1}
\end{aligned}
$$

we find immediately Eq. (1.5).

Thus our results coincide with the result found by W. Pohl for plane convex curves. In Sect. 2, we derive an intermediate result and we find that $J_{A B}$ can be expressed in a simple way in terms of functions $\mathscr{A}_{A}[p], \mathscr{A}_{B}[p], \mathscr{B}_{A}[p]$ and $\mathscr{B}_{B}[p]$ of a variable $p$ which can be considered as the magnitude of a "wave-vector".

The final result is derived in Sect. 3 and a few remarks are made in Sect. 4.

A different derivation and a further generalization have been found by Duplantier [4]. These will be described in another article.

\section{Calculation of $J_{A B}$; Representation of $J_{A B}$ in "Wave-Vector Space"}

The linking number $I_{A B}$ of two curves $A$ and $B$ can be written

$$
I_{A B}=\frac{1}{4 \pi} \int_{A} \int_{B}\left(d \mathbf{r}_{A}, d \mathbf{r}_{B}, \nabla_{A}\left(\frac{1}{r_{A B}}\right)\right) .
$$

On the other hand, we know that

$$
\frac{1}{r}=\frac{1}{2 \pi^{2}} \int d^{3} p \frac{e^{i \mathbf{p} \cdot \mathbf{r}}}{p^{2}}
$$

(remember that $\Delta(1 / r)=-4 \pi \delta(\mathbf{r})$ ).

We may then write

$$
I_{A B}=\frac{i}{8 \pi^{3}} \int d^{3} p \iint_{A}\left(d \mathbf{r}_{A}, d \mathbf{r}_{B}, \mathbf{p}\right) \frac{e^{i \mathbf{p} \cdot\left(\mathbf{r}_{A}-\mathbf{r}_{B}\right)}}{p^{2}},
$$

and therefore we have

(see Fig. 2).

$$
\begin{aligned}
I_{A B}^{2}= & -\frac{1}{(2 \pi)^{6}} \int \frac{d^{3} p}{p^{2}} \frac{d^{3} q}{q^{2}} e^{i \mathbf{p} \cdot\left(\mathbf{r}_{A 1}-\mathbf{r}_{B 1}\right)} \\
& \cdot e^{i \mathbf{q} \cdot\left(\mathbf{r}_{A 2}-\mathbf{r}_{B 2}\right)}\left(d \mathbf{r}_{A 1}, d \mathbf{r}_{B 1}, \mathbf{p}\right)\left(d \mathbf{r}_{A 2}, d \mathbf{r}_{B 2}, \mathbf{q}\right)
\end{aligned}
$$




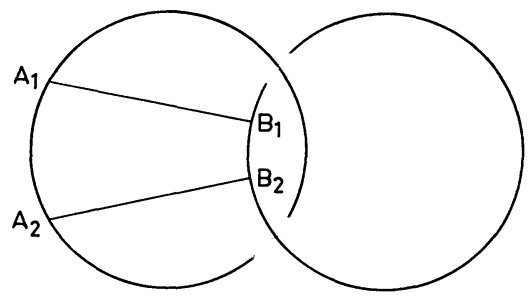

Fig. 2. The curves $A$ and $B$

Now let us translate curve $B$ with respect to curve $A$ by a vector $\rho$. We obtain in this way a linking number $I_{A B}(\rho)$. To obtain $I_{A B}(\rho)$, we make the transformation

$$
\begin{aligned}
& \mathbf{r}_{B 1} \rightarrow \mathbf{r}_{B 1}+\boldsymbol{\rho} \\
& \mathbf{r}_{B 2} \rightarrow \mathbf{r}_{B 2}+\boldsymbol{\rho}
\end{aligned}
$$

in the preceding formula. In this way, we obtain in the integrand an extra factor $e^{-i(\mathbf{p}+\mathbf{q}) \cdot \boldsymbol{\rho}}$.

Let us now calculate $\int d^{3} \rho I_{A B}^{2}(\rho)$.

Since

$$
\int d^{3} \rho e^{-i(\mathbf{p}+\mathbf{q}) \cdot \boldsymbol{\rho}}=(2 \pi)^{3} \delta(\mathbf{p}+\mathbf{q}),
$$

we obtain, after integration with respect to $\mathbf{q}$,

$$
\begin{aligned}
\int d^{3} \rho I_{A B}^{2}(\boldsymbol{\rho})= & \frac{1}{(2 \pi)^{3}} \int \frac{d^{3} p}{p^{4}} e^{i \mathbf{p} \cdot\left(\mathbf{r}_{A 1}-\mathbf{r}_{A 2}\right)} \\
& \cdot e^{-i \mathbf{p} \cdot\left(\mathbf{r}_{B 1}-\mathbf{r}_{B 2}\right)}\left(d \mathbf{r}_{A 1}, d \mathbf{r}_{B 1}, \mathbf{p}\right)\left(d \mathbf{r}_{A 2} d \mathbf{r}_{B 2}, \mathbf{p}\right) .
\end{aligned}
$$

Now, we have

$$
\left(d \mathbf{r}_{A 1}, d \mathbf{r}_{B 1}, \mathbf{p}\right)\left(d \mathbf{r}_{A 2}, d \mathbf{r}_{B 2}, \mathbf{p}\right)=\left|\begin{array}{ccc}
d x_{A 1} & d y_{A 1} & d z_{A 1} \\
d x_{B 1} & d y_{B 1} & d z_{B 1} \\
p_{x} & p_{y} & p_{z}
\end{array}\right| \quad\left|\begin{array}{ccc}
d x_{A 2} & d x_{B 2} & p_{x} \\
d y_{A 2} & d y_{B 2} & p_{y} \\
d z_{A 2} & d z_{B 2} & p_{z}
\end{array}\right|
$$

and therefore

$$
\left(d \mathbf{r}_{A 1}, d \mathbf{r}_{B 1}, \mathbf{p}\right)\left(d \mathbf{r}_{A 2}, d \mathbf{r}_{B 2}, \mathbf{p}\right)=\left|\begin{array}{ccc}
d \mathbf{r}_{A 1} \cdot d \mathbf{r}_{A 2} & d \mathbf{r}_{A 1} \cdot d \mathbf{r}_{B 2} & d \mathbf{r}_{A 1} \cdot \mathbf{p} \\
d \mathbf{r}_{B 1} \cdot d \mathbf{r}_{A 2} & d \mathbf{r}_{B 1} \cdot d \mathbf{r}_{B 2} & d \mathbf{r}_{B 1} \cdot \mathbf{p} \\
d \mathbf{r}_{A 2} \cdot \mathbf{p} & d \mathbf{r}_{B 2} \cdot \mathbf{p} & p^{2}
\end{array}\right|
$$

When the preceding determinant is introduced in Eq. (2.4), simplifications occur. We note that

$$
\int_{C} e^{i \mathbf{p} \cdot \mathbf{r}}(d \mathbf{r} \cdot \mathbf{p})=-i \int_{C} d \mathbf{r} \cdot \boldsymbol{\nabla}\left(e^{i \mathbf{p} \cdot \mathbf{r}}\right)=0
$$

the integration being made along any closed curve $C$. On the other hand, $d \mathbf{r}_{A 1}$ occurs only in the first row, $d \mathbf{r}_{B 1}$ only in the second row. Similarly $d \mathbf{r}_{A 2}$ occurs 
only in the first column, $d \mathbf{r}_{B 2}$ only in the second column. This remark shows that in Eq. (2.4), we may replace the preceding determinant by a simplified determinant

$$
\begin{aligned}
& \left|\begin{array}{ccc}
d \mathbf{r}_{A 1} \cdot d \mathbf{r}_{A 2} & d \mathbf{r}_{A 1} \cdot d \mathbf{r}_{B 2} & 0 \\
d \mathbf{r}_{B 1} \cdot d \mathbf{r}_{A 2} & d \mathbf{r}_{B 1} \cdot d \mathbf{r}_{B 2} & 0 \\
0 & 0 & p^{2}
\end{array}\right| \\
& \quad=p^{2}\left[\left(d \mathbf{r}_{A 1} \cdot d \mathbf{r}_{A 2}\right)\left(d \mathbf{r}_{B 1} \cdot d \mathbf{r}_{B 2}\right)-\left(d \mathbf{r}_{A 1} \cdot d \mathbf{r}_{B 2}\right)\left(d \mathbf{r}_{B 1} \cdot d \mathbf{r}_{A 2}\right)\right]
\end{aligned}
$$

Let us introduce this expression in Eq. (2.5). After permuting $B 1$ and $B 2$, we obtain $^{2}$

$$
\begin{aligned}
\int d^{3} \rho I_{A B}^{2}(\boldsymbol{\rho})= & \frac{1}{(2 \pi)^{3}} \int \frac{d^{3} p}{p^{2}} e^{i \mathbf{p}\left(\mathbf{r}_{A 1}-\mathbf{r}_{A 2}\right)} e^{i \mathbf{p}\left(\mathbf{r}_{B 1}-\mathbf{r}_{B 2}\right)} \\
& \cdot\left[\left(d r_{A 1}^{j} d r_{A 2}^{j}\right)\left(d r_{B 1}^{k} d r_{B 2}^{k}\right)-\left(d r_{A 1}^{j} d r_{A 2}^{k}\right)\left(d r_{B 1}^{j} d r_{B 2}^{k}\right)\right] .
\end{aligned}
$$

Now by changing the orientations of $B$ and $A$ we obtain $\int d^{3} \rho I_{A B}^{2}(\rho, \mathcal{O})$ and by averaging over all orientations we obtain

$$
\begin{aligned}
J_{A B} & =\int d^{3} \rho\left\langle I_{A B}^{2}(\boldsymbol{\rho}, \mathcal{O})\right\rangle \\
& =\frac{1}{(2 \pi)^{3}} \int \frac{d^{3} p}{p^{2}}\left[\mathscr{A}_{A}[p] \mathscr{A}_{B}[p]-\mathscr{B}_{A}^{j k}[p] \mathscr{B}_{B}^{j k}[p]\right],
\end{aligned}
$$

where $\mathscr{A}[p]$ and $\mathscr{B}[p]$ for the curve $C$ are defined by double integrals averaged over all orientations of $C$ in space.

$$
\begin{aligned}
\mathscr{A}[p] & =\left\langle\iint e^{i \mathbf{p}\left(\mathbf{r}_{1}-\mathbf{r}_{2}\right)}\left(d \mathbf{r}_{1} \cdot d \mathbf{r}_{2}\right)\right\rangle \\
\mathscr{B}^{i j}[p] & =\left\langle\iint e^{i \mathbf{p}\left(\mathbf{r}_{1}-\mathbf{r}_{2}\right)}\left(d r_{1}^{i} d r_{2}^{j}\right)\right\rangle .
\end{aligned}
$$

We note that

$$
\mathscr{B}^{j j}[p]=\mathscr{A}[p]
$$

and that

$$
p^{j} \mathscr{B}^{j k}[p]=0
$$

[see Eq. (2.6)].

On the other hand, it will be convenient to introduce the function $\mathscr{B}[p]$

$$
\begin{aligned}
\mathscr{B}[p] & =-i\left\langle\iint e^{i \mathbf{p}\left(\mathbf{r}_{1}-\mathbf{r}_{2}\right)}\left(d \mathbf{r}_{1}, d \mathbf{r}_{2}, \frac{\mathbf{p}}{p}\right)\right\rangle \\
& =\frac{1}{p}\left\langle\iint\left(d \mathbf{r}_{1}, d \mathbf{r}_{2}, \nabla_{2}\right) e^{i \mathbf{p}\left(\mathbf{r}_{1}-\mathbf{r}_{2}\right)}\right\rangle .
\end{aligned}
$$

Now it is possible to express $\mathscr{B}^{j k}[p]$ in terms of the scalar functions $\mathscr{A}[p]$ and $\mathscr{B}[p]$. In fact, group theory shows that $\mathscr{B}^{j k}[p]$ must be of the form

$$
\mathscr{B}^{j k}[p]=\delta^{j k} f(p)+p^{j} p^{k} g(p)+\varepsilon^{j k \ell} p^{\ell} h(p) .
$$

2 Summation over repeated indices is implicit in (2.8) and subsequent equations 
Here $\varepsilon^{j k \ell}=+1$ if $(j, k, \ell)$ is an even permutation of $(1,2,3), \varepsilon^{j k \ell}=-1$ if $(j, k, \ell)$ is an odd permutation of $(1,2,3)$ and $\varepsilon^{j k \ell}=0$ otherwise.

Let us calculate $f(p), g(p)$ and $h(p)$. Eqs. (2.12) and (2.13) give

and therefore

$$
\begin{aligned}
3 f(p)+p^{2} g(p) & =\mathscr{A}[p] \\
f(p)+p^{2} g(p) & =0,
\end{aligned}
$$

$$
\begin{aligned}
f(p) & =\frac{1}{2} \mathscr{A}[p] \\
p^{2} g(p) & =-\frac{1}{2} \mathscr{A}[p] .
\end{aligned}
$$

On the other hand, from Eqs. (2.14), (2.11) and (2.15) we deduce

$$
\begin{aligned}
\mathscr{B}[p] & =-\frac{i}{p} \varepsilon^{j k \ell} \mathscr{B}^{j k}[p] p^{\ell} \\
& =-\frac{i}{p} \varepsilon^{j k \ell} \varepsilon^{j k m} p^{\ell} p^{m} h(p) \\
& =-2 i p h(p),
\end{aligned}
$$

and therefore

$$
h(p)=+\frac{i}{2 p} \mathscr{B}[p]
$$

Now let us calculate the product $\mathscr{B}_{A}^{j k}[p] \mathscr{B}_{B}^{j k}[p]$; starting from Eq. (2.15), we may write

$$
\begin{aligned}
\mathscr{B}_{A}^{j k}[p] \mathscr{B}_{B}^{j k}[p]= & 3 f_{A}(p) f_{B}(p)+p^{2}\left[f_{A}(p) g_{B}(p)+g_{A}(p) f_{B}(p)\right] \\
& +p^{4} g_{A}(p) g_{B}(p)+2 p^{2} h_{A}(p) h_{B}(p),
\end{aligned}
$$

and with the help of Eqs. (2.16) and (2.18), we get

$$
\mathscr{B}_{A}^{j k}[p] \mathscr{B}_{B}^{j k}[p]=\frac{1}{2} \mathscr{A}_{A}[p] \mathscr{A}_{B}[p]-\frac{1}{2} \mathscr{B}_{B}[p] \mathscr{B}_{B}[p]
$$

where the functions $\mathscr{A}[p]$ and $\mathscr{B}[p]$ depend only on the length $p$ of $\mathbf{p}$.

Let us bring (2.19) into Eq. (2.9).

We obtain the result

$$
J_{A B}=\frac{1}{(2 \pi)^{2}} \int_{0}^{\infty} d p\left\{\mathscr{A}_{A}[p] \mathscr{A}_{B}[p]+\mathscr{B}_{A}[p] \mathscr{B}_{B}[p]\right\}
$$

On the other hand, in the definitions of $\mathscr{A}[p]$ and $\mathscr{B}[p]$, instead of averaging over the orientations of the curve under consideration, we may average over the orientation of p. Thus, Eq. (2.10) gives

$$
\mathscr{A}[p]=\iint\left(d \mathbf{r}_{1} \cdot d \mathbf{r}_{2}\right) \frac{\sin \left(p r_{12}\right)}{p r_{12}}
$$


and Eq. (2.14) gives

$$
\begin{aligned}
\mathscr{B}[p] & =\iint\left(d \mathbf{r}_{1}, d \mathbf{r}_{2}, \nabla_{2}\right) \frac{\sin \left(p r_{12}\right)}{p^{2} r_{12}} \\
& =\iint\left(d \mathbf{r}_{1}, d \mathbf{r}_{2}, \mathbf{r}_{12}\right)\left[\frac{\cos \left(p r_{12}\right)}{p r_{1}^{2}}-\frac{\sin \left(p r_{12}\right)}{p^{2} r_{12}^{3}}\right] .
\end{aligned}
$$

Equations (2.20), (2.21), and (2.22) constitute the intermediate results announced in the introduction.

\section{Representation of $J_{A B}$ in "Real Space"}

In the preceding section, $J_{A B}$ has been expressed as an integral over a variable $p$ which has the dimension of a wave vector (the inverse of a length). By performing a Fourier transform, it is possible to express $J_{A B}$ as an integral over a length $r$. The transformation is not unique and various results can be obtained. A simple way of obtaining the simplest result consists of replacing $\mathscr{A}[p]$ and $\mathscr{B}[p]$ in Eq. (2.20) by their formal expressions (2.21) and (2.22) and integrating with respect to $p$. We may set $r_{A}=\left|\mathbf{r}_{A 1}-\mathbf{r}_{A 2}\right|, r_{B}=\left|\mathbf{r}_{B 1}-\mathbf{r}_{B 2}\right|$ and we shall use the following identities, which are derived in the appendix:

$$
\begin{aligned}
& \int_{0}^{\infty} d p \frac{\sin p r_{A}}{p r_{A}} \frac{\sin p r_{B}}{p r_{B}}=\frac{\pi}{2} \int_{0}^{\infty} d r \frac{1}{r^{2}} \theta\left(r-r_{A}\right) \theta\left(r-r_{B}\right), \\
& \int_{0}^{\infty} d p\left(\frac{\cos p r_{A}}{p r_{A}^{2}}-\frac{\sin p r_{A}}{p^{2} r_{A}^{3}}\right)\left(\frac{\cos p r_{B}}{p r_{B}^{2}}-\frac{\sin p r_{B}}{p^{2} r_{B}^{3}}\right) \\
& =\frac{\pi}{2} \int_{0}^{\infty} d r \frac{1}{r^{4}} \theta\left(r-r_{A}\right) \theta\left(r-r_{B}\right) .
\end{aligned}
$$

This shows immediately that $J_{A B}$ is given by

$$
J_{A B}=\frac{1}{8 \pi} \int_{0}^{\infty} d r\left[\mathscr{A}_{A}(r) \mathscr{A}_{B}(r)+\mathscr{B}_{A}(r) \mathscr{B}_{B}(r)\right]
$$

where $\mathscr{A}(r)$ is derived from $(2.21)$ and (3.1)

$$
\mathscr{A}(r)=\iint\left(d \mathbf{r}_{1} \cdot d \mathbf{r}_{2}\right) \frac{1}{r} \theta\left(r-r_{12}\right)
$$

and $\mathscr{B}(r)$ from (2.22) and (3.2)

$$
\mathscr{B}(r)=\iint\left(d \mathbf{r}_{1}, d \mathbf{r}_{2}, \mathbf{r}_{12}\right) \frac{1}{r^{2}} \theta\left(r-r_{12}\right) .
$$

Thus we find exactly the results announced in the introduction [see Eqs. (1.8) and (1.9)]. 


\section{Remarks}

Obviously the function $\mathscr{A}(r)$ associated with a curve $C$ is never identically zero, since $\mathscr{A}(0)=2 L$ where $L$ is the length of the curve. However, as was noted in the introduction, $\mathscr{B}(r)$ may vanish for all $r$.

Let $C^{\prime}$ be the mirror image of $C$. It is easy to see that

$$
\begin{aligned}
& \mathscr{A}_{C^{\prime}}(r)=\mathscr{A}_{C}(r) \\
& \mathscr{B}_{C^{\prime}}(r)=-\mathscr{B}_{C}(r) .
\end{aligned}
$$

Therefore, if $C$ coincides with $C^{\prime}, \mathscr{B}_{C}(r)=0$. However, it is possible to show the existence of curves $C$ for which $\mathscr{B}(r)$ does not vanish. To establish this fact, it is sufficient to find a curve $C$ for which

$$
\frac{\partial}{\partial r}\left[r^{2} \mathscr{B}(r)\right] \equiv \iint\left(d \mathbf{r}_{1}, d \mathbf{r}_{2}, \mathbf{r}_{12}\right) \delta\left(r-r_{12}\right) \neq 0 .
$$

Consider now the curve $C$ shown on Fig. 3. The main contribution is due to the central part, which can be made as long as we wish. Then, if we choose for $r$ a value which is much smaller than the radius $R$ and the pitch $H$, we see that the contribution of the central part comes only from the helix and has a definite sign.

Equations (4.1) show that

$$
J_{C C^{\prime}} \leqq J_{C C}
$$

Thus, in general, if $C^{\prime}$ does not coincide with $C$, we have

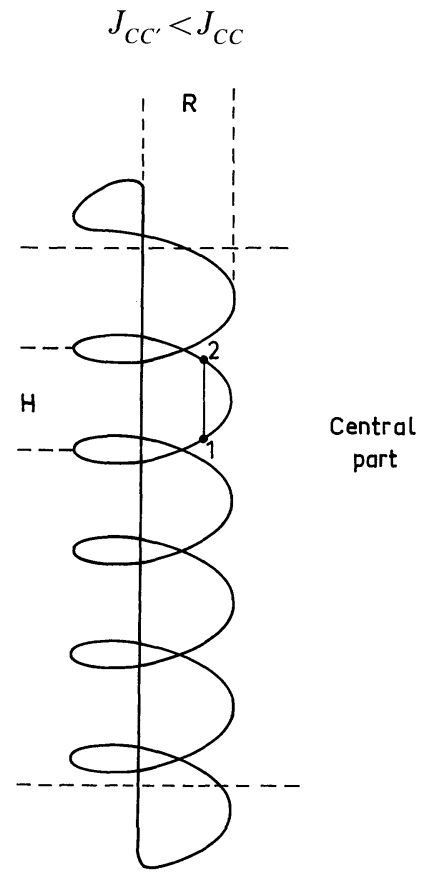

Fig. 3. A curve $C$ for which $\mathscr{B}(r)$ does not vanish 


\section{Appendix}

The identities (3.1) and (3.2) are derived will the help of the formulas

$$
\frac{\pi}{2}=\int_{0}^{\infty} d x \frac{\sin x}{x}=\int_{0}^{\infty} d x \frac{1-\cos x}{x^{2}}=2 \int_{0}^{\infty} d x \frac{x-\sin x}{x^{3}}=6 \int_{0}^{\infty} d x \frac{\cos x-1+x^{2} / 2}{x^{4}} .
$$

First Identity

$$
\begin{aligned}
I_{a} & =\int_{0}^{\infty} d p \frac{\sin p r_{A}}{p r_{A}} \frac{\sin p r_{B}}{p r_{B}} \\
& =\frac{1}{2 r_{A} r_{B}} \int_{0}^{\infty} d p\left[\frac{\cos p\left(r_{A}-r_{B}\right)-\cos p\left(r_{A}+r_{B}\right)}{p^{2}}\right] \\
& =\frac{1}{2 r_{A} r_{B}} \frac{\pi}{2}\left[-\left|r_{A}-r_{B}\right|+\left|r_{A}+r_{B}\right|\right] \\
& =\frac{\pi}{2} \operatorname{Min}\left(\frac{1}{r_{A}}, \frac{1}{r_{B}}\right) .
\end{aligned}
$$

Therefore, we have also

$$
I_{a}=\frac{\pi}{2} \int_{0}^{\infty} d r \frac{1}{r^{2}} \theta\left(r-r_{A}\right) \theta\left(r-r_{B}\right)
$$

Second Identity

$$
I_{b}=\int_{0}^{\infty} d p\left[\frac{\cos p r_{A}}{p r_{A}^{2}}-\frac{\sin p r_{A}}{p^{2} r_{A}^{3}}\right]\left[\frac{\cos p r_{B}}{p r_{B}^{2}}-\frac{\sin p r_{B}}{p^{2} r_{B}^{3}}\right] .
$$

We note that

$$
I_{b}=\frac{1}{r_{A} r_{B}} \frac{\partial^{2}}{\partial r_{A} \partial r_{B}} J_{b}
$$

and then find

$$
\begin{aligned}
J_{b}= & \int_{0}^{\infty} d p\left(\frac{\sin p r_{A}}{p^{2} r_{A}}-\frac{1}{p}\right)\left(\frac{\sin p r_{B}}{p^{2} r_{B}}-\frac{1}{p}\right) \\
= & \int_{0}^{\infty} d p\left[\frac{\cos p\left(r_{A}-r_{B}\right)-\cos p\left(r_{A}+r_{B}\right)}{2 r_{A} r_{B} p^{4}}-\frac{1}{p^{2}}\right] \\
& +\int_{0}^{\infty} d p\left[\frac{2}{p^{2}}-\frac{\sin p r_{A}}{p^{3} r_{A}}-\frac{\sin p r_{B}}{p^{3} r_{B}}\right] \\
= & \frac{1}{2 r_{A} r_{B}} \frac{\pi}{12}\left[\left|r_{A}-r_{B}\right|^{3}-\left(r_{A}+r_{B}\right)^{3}\right]+\frac{\pi}{4}\left[r_{A}+r_{B}\right] .
\end{aligned}
$$

This can be rewritten as

$$
J_{b}=\frac{\pi}{12}\left[\left(3 r_{B}-\frac{r_{B}^{2}}{r_{A}}\right) \theta\left(r_{A}-r_{B}\right)+\left(3 r_{A}-\frac{r_{A}^{2}}{r_{B}}\right) \theta\left(r_{B}-r_{A}\right)\right]
$$


and we then derive $I_{b}$ :

Therefore, we have also

$$
\begin{aligned}
I_{b}=\frac{1}{r_{A} r_{B}} \frac{\partial^{2}}{\partial r_{A} \partial r_{B}} I_{b} & =\frac{\pi}{6}\left[\frac{1}{r_{A}^{3}} \theta\left(r_{A}-r_{B}\right)+\frac{1}{r_{B}^{3}} \theta\left(r_{B}-r_{A}\right)\right] \\
& =\frac{\pi}{6} \operatorname{Min}\left(\frac{1}{r_{A}^{3}}, \frac{1}{r_{B}^{3}}\right) .
\end{aligned}
$$

$$
I_{b}=\frac{\pi}{2} \int_{0}^{\infty} d r \frac{1}{r^{4}} \theta\left(r-r_{A}\right) \theta\left(r-r_{B}\right)
$$

\section{References}

1. Rolfsen, D.: Knots and Links. Mathematics Lecture Series, Vol. 7, p. 132. Berkeley, CA: Publish or Perish, Inc. 1976

2. Alexandroff, P. and Hopf, H.: Topologie I. Berlin: Springer 1935

3. Pohl, W.: International Symposium in honour of N.H. Kuiper, Utrecht 1980. In: Lecture Notes in Mathematics. Berlin, Heidelberg, New York: Springer (to be published)

4. Duplantier, B.: Linking numbers, contracts, and mutal inductances of a Random set of closed curves. Commun. Math. Phys. (to appear)

Communicated by J.L. Lebowitz

Received February 17, 1981 
